\title{
Optimal Detector Randomization in Cognitive Radio Systems in the Presence of Imperfect Sensing Decisions
}

\author{
Ahmet Dundar Sezer, Sinan Gezici, and Mustafa Cenk Gursoy
}

\begin{abstract}
In this study, optimal detector randomization is developed for secondary users in a cognitive radio system in the presence of imperfect spectrum sensing decisions. It is shown that the minimum average probability of error can be achieved by employing no more than four maximum a-posteriori probability (MAP) detectors at the secondary receiver. Optimal MAP detectors and generic expressions for their average probability of error are derived in the presence of possible sensing errors. Also, sufficient conditions are presented related to improvements due to optimal detector randomization.
\end{abstract}

Index Terms-Cognitive radio, spectrum sensing, detector randomization, probability of error.

\section{INTRODUCTION}

$\mathbf{I}$ $\mathrm{N}$ cognitive radio systems, spectrum sensing is one of the crucial tasks to be performed by secondary users in order to limit the interference to primary users. Therefore, various spectrum sensing methods have been proposed in the literature such as matched filtering, energy detection, and cyclostationary detection [1]. Once secondary users make a sensing decision, they adapt their communication parameters accordingly. Specifically, they perform communications when the channel is sensed as idle (i.e., no primary user activity is detected), whereas they either do not transmit at all or transmit at a reduced power when the channel is sensed as busy [1]. In practical systems, sensing decisions of secondary users are never perfect; hence, there can be cases in which the sensing decision is idle (busy) but primary user activity actually exists (does not exist). In most of the studies in the literature, communications systems of secondary users are designed independently of the sensing decision, or, the sensing decisions are considered as perfect. However, the optimal design of secondary systems requires the consideration of imperfect sensing decisions. In [2], interactions between spectrum sensing and channel estimation are studied, and the dependence of channel estimators on sensing decisions is investigated. In addition, an approach is proposed in [3] to perform spectrum sensing and data transmission simultaneously for optimizing the sensing time and the throughput of the secondary system.

In this study, the aim is to design the optimal secondary communications system in the presence of detector randomization by taking imperfect channel sensing decisions into account. Detector randomization is a technique to employ multiple detectors at the receiver with certain probabilities (certain fractions of time) [4], [5]. By adapting the transmitted power level according to the employed detector at the receiver, performance improvements can be achieved via

Manuscript received October 30, 2013. The associate editor coordinating the review of this letter and approving it for publication was D. Cabric.

A. D. Sezer and S. Gezici are with the Dept. of Electrical and Electronics Eng., Bilkent University, Ankara, Turkey, e-mails: \{adsezer, gezici\}@ee.bilkent.edu.tr).

M. C. Gursoy is with the Dept. of Electrical Eng. and Computer Science, Syracuse University, Syracuse, NY, 13244, USA.

Digital Object Identifier 10.1109/LCOMM.2013.120713.132423 detector randomization (i.e., via switching between multiple transmit power-detector pairs). As investigated in [4], [5], benefits of detector randomization are observed commonly in non-Gaussian channels. By noting that secondary users in cognitive radio systems experience non-Gaussian channels in practice due to imperfect sensing decisions, we propose the use of detector randomization for the design of secondary communications systems. The main contributions of this study are as follows: (i) Detector randomization is studied for cognitive radio systems for the first time; (ii) optimal detector randomization is developed in the presence of imperfect sensing decisions; (iii) optimal MAP detectors are derived and generic probability of error expressions are obtained in the presence of possible sensing errors.

\section{Optimal Detector Randomization in the PRESENCE OF CHANNEL SENSING ERRORS}

Consider a cognitive radio system in which secondary users first sense the channel in order to identify whether the channel is being utilized by primary users. Let $\mathcal{H}_{0}$ and $\mathcal{H}_{1}$ represent the hypotheses that correspond to the absence and presence of primary user activity, respectively. In addition, let $\hat{\mathcal{H}}_{1}$ denote the event in which the secondary user decides that primary user activity exists in the channel; i.e., declares $\mathcal{H}_{1}$ as the true hypothesis. Similarly, let $\hat{\mathcal{H}}_{0}$ denote the event in which $\mathcal{H}_{0}$ is declared as the true hypothesis by the secondary user. Note that the true underlying hypothesis can be either $\mathcal{H}_{0}$ or $\mathcal{H}_{1}$.

After the channel sensing phase, cognitive secondary users start digital communications. Specifically, the secondary transmitter sends information carrying signals to the secondary receiver in a certain manner depending on the channel sensing decision. It is assumed that the secondary radio channel is subject to slow frequency-flat fading. Then, depending on the channel sensing decision and the true state of the channel (i.e., the presence and absence of primary user activity), the following four scenarios exist:

$$
\begin{aligned}
& \left(\mathcal{H}_{1}, \hat{\mathcal{H}}_{1}\right): x=h \sqrt{P_{1}} d+n+s \\
& \left(\mathcal{H}_{1}, \hat{\mathcal{H}}_{0}\right): x=h \sqrt{P_{0}} d+n+s \\
& \left(\mathcal{H}_{0}, \hat{\mathcal{H}}_{1}\right): x=h \sqrt{P_{1}} d+n \\
& \left(\mathcal{H}_{0}, \hat{\mathcal{H}}_{0}\right): x=h \sqrt{P_{0}} d+n
\end{aligned}
$$

where $\left(\mathcal{H}_{i}, \hat{\mathcal{H}}_{j}\right)$ denotes the scenario in which the sensing decision is $\hat{\mathcal{H}}_{j}$ while the true hypothesis is $\mathcal{H}_{i}$, and $P_{i}$ denotes the power level of the information symbol when the sensing decision is $\hat{\mathcal{H}}_{i}{ }^{1}$ Also, $x$ is the observation at the receiver of the secondary user, $h$ denotes the fading coefficient in the channel between the secondary transmitter and receiver, $n$ denotes the zero-mean complex Gaussian noise with variance

${ }^{1}$ For the theoretical investigations in this study, we consider generic values for $P_{0}$ and $P_{1}$. For example, if no secondary communication is performed when the channel is sensed as busy, then we can set $P_{1}=0$. 


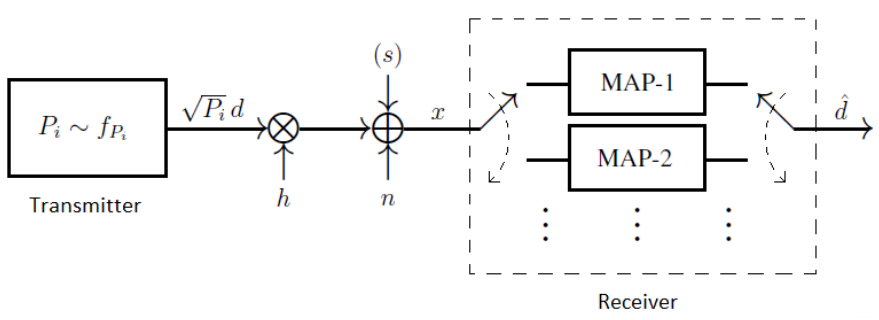

Fig. 1. Basedband model of the communications system for the secondary users. The secondary transmitter generates a signal, the power $P_{i}$ of which is determined according to the PDF $f_{P_{i}}$ for $i \in\{0,1\}$. The information signal, $\sqrt{P_{i}} d$ is multiplied with the complex channel coefficient $h$, and it is corrupted by additive noise $n$. Also, if primary users exist, their faded signals, denoted by $s$, interfere with the desired signal.

$\sigma_{n}^{2}, s$ is the sum of the faded primary users' signals arriving at the secondary receiver, and $d$ denotes the complex information symbol. Without loss of generality, it is assumed that $\mathbb{E}\left\{|d|^{2}\right\}=1$. Considering $M$-ary modulation, the complex information symbol $d$ takes values from set $\left\{d_{0}, d_{1}, \ldots, d_{M-1}\right\}$. Furthermore, it is assumed that the channel coefficient $h$ is known at the secondary receiver; i.e., channel estimation is performed perfectly before the communications start.

It is noted that in the presence of primary user activity, the additive disturbance is noise plus the primary users' received sum signal, i.e., $n+s$, as in (1) and (2), while only additive noise is present when the channel is not occupied by the primary users. Since errors are possible in channel sensing, the true state of the channel (busy or idle) and consequently the statistics of the additive disturbance are not perfectly known by the secondary receiver. Hence, the optimal communication system needs to be designed in the presence of such sensing errors and ambiguities.

We consider a secondary communications system as in Fig. 1, where the secondary transmitter can randomize the power levels, $P_{0}$ and $P_{1}$ in (1)-(4), and the secondary receiver can perform a corresponding randomization (time-sharing) among multiple MAP detectors. The power levels $P_{0}$ and $P_{1}$ are generated according to PDFs $f_{P_{0}}$ and $f_{P_{1}}$, respectively, depending on the sensing decision. It is assumed that for each possible power level used by the secondary transmitter, the secondary receiver can employ the corresponding optimal MAP detector for that power level. Hence, there exist as many MAP detectors at the secondary receiver as the number of different transmit power levels. Although we start with such a generic formulation in order to obtain the optimal error performance that can be achieved by the secondary system, we show in the following that no more than four MAP detectors are necessary for obtaining the overall optimal solution.

Remark 1: MAP detectors are employed in Fig. 1 since they minimize the average probability of error among all possible detectors.

Based on the formulation in (1)-(4) and the system model in Fig. 1, the aim is to find the optimal power distributions for $P_{0}$ and $P_{1}$ in order to minimize the average error probability of the secondary system under the following average and peak power constraints:

$$
\mathbb{E}\left\{P_{i}\right\} \leq P_{\mathrm{av}, i} \text { and } P_{i} \leq P_{\mathrm{pk}, i} \text { for } i \in\{0,1\}
$$

where $P_{\mathrm{av}, i}$ and $P_{\mathrm{pk}, i}$ are the limits on the average and peak powers, respectively. Note that the average power constraints in (5) also imply limits on the average transmit power at the secondary transmitter and on the average interference power to primary users.

Let $\mathbb{P}_{\mathrm{e}, i}$ denote the average probability of error for the secondary receiver when the sensing decision is $\hat{\mathcal{H}}_{i}$, where $i \in\{0,1\}$. Then, the proposed optimal detector randomization problem can be formulated under the constraints in (5) as follows:

$$
\begin{aligned}
\min _{f_{P_{0}}, f_{P_{1}}} & \operatorname{Pr}\left\{\hat{\mathcal{H}}_{0}\right\} \mathbb{P}_{\mathrm{e}, 0}+\operatorname{Pr}\left\{\hat{\mathcal{H}}_{1}\right\} \mathbb{P}_{\mathrm{e}, 1} \\
\text { subject to } & \mathbb{E}\left\{P_{i}\right\} \leq P_{\mathrm{av}, i}, P_{i} \leq P_{\mathrm{pk}, i} \text { for } i \in\{0,1\} .
\end{aligned}
$$

where $\operatorname{Pr}\left\{\hat{\mathcal{H}}_{i}\right\}$ is the probability that the sensing decision is $\hat{\mathcal{H}}_{i}$, and $f_{P_{i}}$ denotes the PDF of the power parameter $P_{i}$ for $i \in\{0,1\}$.

Due to the structure of the optimization problem in (6), the optimal power distributions can be obtained separately for $P_{0}$ and $P_{1}$ as follows:

$$
\min _{f_{P_{i}}} \mathbb{P}_{\mathrm{e}, i} \text { subject to } \mathbb{E}\left\{P_{i}\right\} \leq P_{\mathrm{av}, i}, P_{i} \leq P_{\mathrm{pk}, i}
$$

for $i \in\{0,1\}$. In order to obtain a solution of the optimization problem in (7), $\mathbb{P}_{\mathrm{e}, i}$ is evaluated for optimal MAP detectors in the following proposition (cf. Remark 1).

Proposition 1: Consider a scenario in which the sensing decision is $\hat{\mathcal{H}}_{i}$. Suppose that the secondary transmitter employs a power randomization strategy according to $P D F f_{P_{i}}$, and the secondary receiver employs the corresponding randomization of MAP detectors. Then, $\mathbb{P}_{\mathrm{e}, i}$ in (7) can be expressed as

$$
\mathbb{P}_{\mathrm{e}, i}=1-\mathbb{E}\left\{\phi_{i}\left(P_{i}\right)\right\}
$$

with

$$
\begin{aligned}
\phi_{i}\left(P_{i}\right) \triangleq & \int \max _{l \in\{0,1, \ldots, M-1\}}\left\{\operatorname { P r } \{ d _ { l } \} \left(\operatorname{Pr}\left\{\mathcal{H}_{0} \mid \hat{\mathcal{H}}_{i}\right\} f\left(x \mid d_{l}, \hat{\mathcal{H}}_{i}, \mathcal{H}_{0}\right)\right.\right. \\
& \left.\left.+\operatorname{Pr}\left\{\mathcal{H}_{1} \mid \hat{\mathcal{H}}_{i}\right\} f\left(x \mid d_{l}, \hat{\mathcal{H}}_{i}, \mathcal{H}_{1}\right)\right)\right\} d x
\end{aligned}
$$

where $\operatorname{Pr}\left\{d_{l}\right\}$ is the prior probability of information symbol $d_{l}, \operatorname{Pr}\left\{\mathcal{H}_{j} \mid \hat{\mathcal{H}}_{i}\right\}$ is the conditional probability of $\mathcal{H}_{j}$ when the sensing decision is $\hat{\mathcal{H}}_{i}$, and $f\left(x \mid d_{l}, \hat{\mathcal{H}}_{i}, \mathcal{H}_{j}\right)$ denotes the conditional PDF of observation $x$ when information symbol $d_{l}$ is sent, the sensing decision is $\hat{\mathcal{H}}_{i}$ and the true hypothesis is $\mathcal{H}_{j}$.

Proof: When the sensing decision is $\hat{\mathcal{H}}_{i}$, the following MAP decision rule is employed in order to estimate the information symbol for a given value of $P_{i}$ :

$$
\hat{d}=d_{k} \text { where } k=\underset{l \in\{0,1, \ldots, M-1\}}{\arg \max } \operatorname{Pr}\left\{d_{l} \mid x, \hat{\mathcal{H}}_{i}\right\} .
$$

Then, the following manipulations can be performed to derive alternative expressions:

$$
\begin{aligned}
& k=\underset{l \in\{0,1, \ldots, M-1\}}{\arg \max } \operatorname{Pr}\left\{d_{l}, \hat{\mathcal{H}}_{i}\right\} f\left(x \mid d_{l}, \hat{\mathcal{H}}_{i}\right) \\
&=\underset{l \in\{0,1, \ldots, M-1\}}{\arg \max } \operatorname{Pr}\left\{d_{l}\right\} f\left(x \mid d_{l}, \hat{\mathcal{H}}_{i}\right) \\
&=\underset{l \in\{0,1, \ldots, M-1\}}{\arg \max } \operatorname{Pr}\left\{d_{l}\right\}\left(\operatorname{Pr}\left\{\mathcal{H}_{0} \mid \hat{\mathcal{H}}_{i}\right\} f\left(x \mid d_{l}, \hat{\mathcal{H}}_{i}, \mathcal{H}_{0}\right)\right. \\
&\left.\quad+\operatorname{Pr}\left\{\mathcal{H}_{1} \mid \hat{\mathcal{H}}_{i}\right\} f\left(x \mid d_{l}, \hat{\mathcal{H}}_{i}, \mathcal{H}_{1}\right)\right)
\end{aligned}
$$

where (11) is obtained from (10) based on Bayes' rule, (12) follows from the independence of $d_{l}$ and $\hat{\mathcal{H}}_{i}$, and (13) is obtained by conditioning on the true hypotheses. 
When the sensing decision is $\hat{\mathcal{H}}_{i}$, the average probability of error for a given value of $P_{i}$ can be expressed as follows:

$$
\begin{aligned}
\mathbb{P}_{\mathrm{e}, i}\left(P_{i}\right)= & 1-\sum_{l=0}^{M-1} \operatorname{Pr}\left\{d_{l}\right\} \operatorname{Pr}\left\{\hat{d}=d_{l} \mid d_{l}, \hat{\mathcal{H}}_{i}\right\} \\
= & 1-\sum_{l=0}^{M-1} \operatorname{Pr}\left\{d_{l}\right\} \int_{\Gamma_{l, i}} f\left(x \mid d_{l}, \hat{\mathcal{H}}_{i}\right) d x \\
= & 1-\sum_{l=0}^{M-1} \int_{\Gamma_{l, i}} \operatorname{Pr}\left\{d_{l}\right\}\left(\operatorname{Pr}\left\{\mathcal{H}_{0} \mid \hat{\mathcal{H}}_{i}\right\} f\left(x \mid d_{l}, \hat{\mathcal{H}}_{i}, \mathcal{H}_{0}\right)\right. \\
& \left.\quad+\operatorname{Pr}\left\{\mathcal{H}_{1} \mid \hat{\mathcal{H}}_{i}\right\} f\left(x \mid d_{l}, \hat{\mathcal{H}}_{i}, \mathcal{H}_{1}\right)\right) d x
\end{aligned}
$$

where $\Gamma_{l, i}$ denotes the decision region for symbol $l$ of the MAP decision rule corresponding to sensing decision $\hat{\mathcal{H}}_{i}$. Based on (13), $\Gamma_{l, i}$ is specified as the set of $x$ for which $\operatorname{Pr}\left\{d_{l}\right\}\left(\operatorname{Pr}\left\{\mathcal{H}_{0} \mid \hat{\mathcal{H}}_{i}\right\} f\left(x \mid d_{l}, \hat{\mathcal{H}}_{i}, \mathcal{H}_{0}\right)+\right.$ $\left.\operatorname{Pr}\left\{\mathcal{H}_{1} \mid \hat{\mathcal{H}}_{i}\right\} f\left(x \mid d_{l}, \hat{\mathcal{H}}_{i}, \mathcal{H}_{1}\right)\right) \quad \geq \quad \operatorname{Pr}\left\{d_{m}\right\}\left(\operatorname{Pr}\left\{\mathcal{H}_{0} \mid \hat{\mathcal{H}}_{i}\right\}\right.$ $\left.f\left(x \mid d_{m}, \hat{\mathcal{H}}_{i}, \mathcal{H}_{0}\right)+\operatorname{Pr}\left\{\mathcal{H}_{1} \mid \hat{\mathcal{H}}_{i}\right\} f\left(x \mid d_{m}, \hat{\mathcal{H}}_{i}, \mathcal{H}_{1}\right)\right), \forall m \neq l$. Therefore, (16) can be stated as

$$
\begin{aligned}
& \mathbb{P}_{\mathrm{e}, i}\left(P_{i}\right)=1-\int \max _{l \in\{0,1, \ldots, M-1\}}\left\{\operatorname { P r } \{ d _ { l } \} \left(\operatorname{Pr}\left\{\mathcal{H}_{0} \mid \hat{\mathcal{H}}_{i}\right\}\right.\right. \\
& \left.\left.\times f\left(x \mid d_{l}, \hat{\mathcal{H}}_{i}, \mathcal{H}_{0}\right)+\operatorname{Pr}\left\{\mathcal{H}_{1} \mid \hat{\mathcal{H}}_{i}\right\} f\left(x \mid d_{l}, \hat{\mathcal{H}}_{i}, \mathcal{H}_{1}\right)\right)\right\} d x .
\end{aligned}
$$

Since the expression in (17) is conditioned on a given value of $P_{i}$, the average probability of error for a power randomization strategy corresponding to PDF $f_{P_{i}}$ can be expressed as the expectation of (17), which results in

$$
\mathbb{P}_{\mathrm{e}, i}=\int f_{P_{i}}(t) \mathbb{P}_{\mathrm{e}, i}(t) d t=1-\mathbb{E}\left\{\phi_{i}\left(P_{i}\right)\right\}
$$

where $\phi_{i}\left(P_{i}\right)$ is as defined in (9). ${ }^{2}$

Proposition 1 provides an explicit expression for the average probabilities of error under both sensing decisions when a generic power randomization strategy (denoted by $f_{P_{0}}$ or $f_{P_{1}}$ ) and the corresponding MAP detectors are employed as shown in Fig. 1. Based on the proposition (specifically, based on the expression in (8)), the optimal detector randomization problems in (7) can be formulated for $i \in\{0,1\}$ as

$$
\max _{f_{P_{i}}} \mathbb{E}\left\{\phi_{i}\left(P_{i}\right)\right\} \text { subject to } \mathbb{E}\left\{P_{i}\right\} \leq P_{\mathrm{av}, i}, P_{i} \leq P_{\mathrm{pk}, i} .
$$

Although it is challenging to obtain a closed-form solution for the optimal $f_{P_{i}}$ in (19), the form of an optimal solution can be obtained based on the arguments similar to those in [6]. Specifically, when $\phi_{i}$ 's are continuous functions and $P_{i}$ 's take values from finite closed intervals (i.e., $\left[0, P_{\mathrm{pk}, i}\right]$ ), it can be shown that an optimal solution to (19) lies at the boundary of the convex hull of set $U$, which is defined as the set of all possible $\left(P_{i}, \phi_{i}\left(P_{i}\right)\right)$ pairs [6]. Therefore, from Carathéodory's theorem [7], an optimal solution can be obtained as the convex combination of at most two different pairs from set $U$. Hence, an optimal solution to (19) can be expressed in the form of

$$
f_{P_{i}}^{\mathrm{opt}}\left(P_{i}\right)=\lambda_{i} \delta\left(P_{i}-P_{i, 1}\right)+\left(1-\lambda_{i}\right) \delta\left(P_{i}-P_{i, 2}\right),
$$

for $i \in\{0,1\}$, where $P_{i, 1}$ and $P_{i, 2}$ are power values within

\footnotetext{
${ }^{2}$ The dependence of $\phi_{i}\left(P_{i}\right)$ in (9) on the value of $P_{i}$ is through the conditional PDFs $f\left(x \mid d_{l}, \hat{\mathcal{H}}_{i}, \mathcal{H}_{0}\right)$ and $f\left(x \mid d_{l}, \hat{\mathcal{H}}_{i}, \mathcal{H}_{1}\right)$ (please see (1)-(4)).
}

$\left[0, P_{\mathrm{pk}, i}\right], \lambda_{i} \in[0,1]$, and $\delta(\cdot)$ denotes the Dirac delta function.

The form of the optimal solution in (20) implies that, for each sensing decision, the secondary transmitter should perform randomization between at most two different power levels and the secondary receiver needs to perform corresponding detector randomization between at most two different MAP detectors. Therefore, the secondary receiver illustrated in Fig. 1 should implement at most four different MAP detectors considering the two possible sensing decisions, which are the absence $\left(\mathcal{H}_{0}\right)$ and presence $\left(\hat{\mathcal{H}}_{1}\right)$ of primary users.

Based on the expression in (20), the solutions of the optimization problems in (19) can be obtained from the following formulation:

$$
\begin{array}{cl}
\max _{\lambda_{i}, P_{i, 1}, P_{i, 2}} & \lambda_{i} \phi_{i}\left(P_{i, 1}\right)+\left(1-\lambda_{i}\right) \phi_{i}\left(P_{i, 2}\right) \\
\text { subject to } & \lambda_{i} P_{i, 1}+\left(1-\lambda_{i}\right) P_{i, 2} \leq P_{\mathrm{av}, i}, \lambda_{i} \in[0,1] \\
& P_{i, 1} \in\left[0, P_{\mathrm{pk}, i}\right], P_{i, 2} \in\left[0, P_{\mathrm{pk}, i}\right]
\end{array}
$$

for $i \in\{0,1\}$. Compared to (19), the problems in (21) are easier to solve since they require a search over three scalar parameters instead of a search over all possible PDFs.

\section{Performance Evaluation and Conclusions}

In order to investigate the error performance of the optimal detector randomization approach in the previous section, consider a scenario in which noise $n$ in (3) and (4) is modeled as zero-mean, circularly symmetric, complex Gaussian noise, and the sum of faded primary signal and noise, $s+n$, in (1) and (2) is modeled as a mixture of complex Gaussian components each with independent real and imaginary parts having equal variances. That is, the PDFs of $n$ and $s+n \triangleq \varepsilon$ are expressed, respectively, as

$$
\begin{aligned}
& p_{n}(x)=\frac{1}{\pi \sigma_{n}^{2}} \exp \left(-\frac{|x|^{2}}{\sigma_{n}^{2}}\right), \\
& p_{\varepsilon}(x)=\sum_{j=1}^{N_{m}} \frac{\nu_{j}}{\pi \sigma_{j}^{2}} \exp \left(-\frac{\left|x-\mu_{j}\right|^{2}}{\sigma_{j}^{2}}\right)
\end{aligned}
$$

where $\sigma_{n}^{2}$ is the variance of noise $n, N_{m}$ is the number of Gaussian components in the mixture $\varepsilon, \mu_{j}$ and $\sigma_{j}^{2}$ are, respectively, the mean and the variance of the $j$ th component in the mixture, and $\sum_{j=1}^{N_{m}} \nu_{j}=1$ with $\nu_{j} \geq 0, \forall j$.

The main motivation for employing the Gaussian mixture model in (23) is that the sum of noise and interference from primary users can accurately be modeled by a non-Gaussian random variable as discussed, e.g., in [8]. In addition, the Gaussian mixture model in (23) is quite generic since it can model various probability density functions via suitable selection of its parameters.

For the simulations, the receiver is assumed to have perfect channel state information (CSI), and $h$ in (1)-(4) is set to 1 without loss of generality. In addition, $\operatorname{Pr}\left\{\mathcal{H}_{0}\right\}=0.75$ and $\operatorname{Pr}\left\{\mathcal{H}_{1}\right\}=0.25$ are employed.

In order to quantify the improvements achieved via the proposed optimal detector randomization approach, the following approaches are considered as well.

Optimal Single Detector in the Presence of Channel Sensing Errors: In this case, no detector randomization is employed, and the optimal MAP detector is obtained by taking the channel sensing errors into account. As this scenario is a special case of the one in Section II when there is only a 


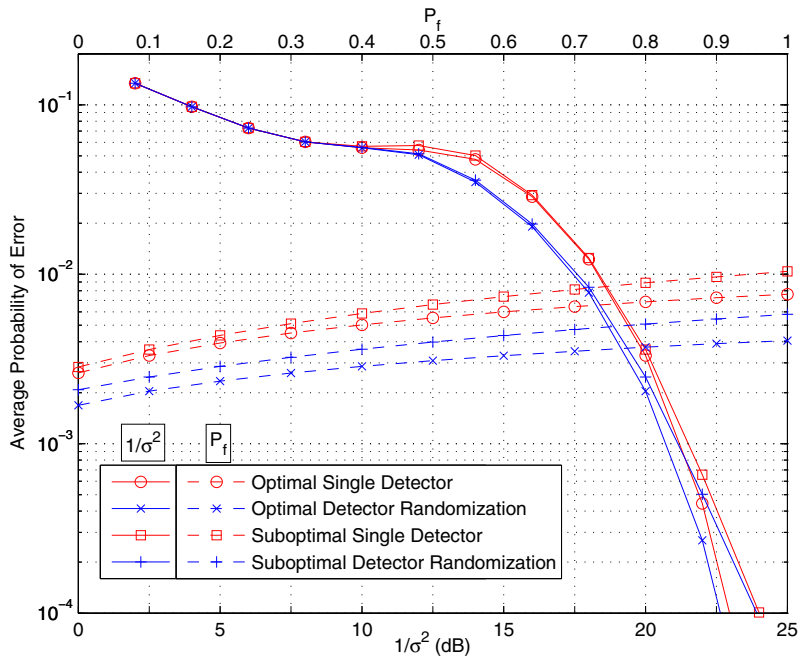

Fig. 2. Average probability of error versus $1 / \sigma^{2}$ and versus $P_{f}=$ $\operatorname{Pr}\left\{\hat{\mathcal{H}}_{1} \mid \mathcal{H}_{0}\right\}$ for different approaches.

single detector, the optimal power values can be obtained as (cf. (19))

$$
\max _{P_{i}} \phi_{i}\left(P_{i}\right) \text { subject to } P_{i} \leq \min \left\{P_{\mathrm{av}, i}, P_{\mathrm{pk}, i}\right\}
$$

for $i \in\{0,1\}$, and the resulting conditional probabilities of error can be calculated from $1-\phi_{i}\left(P_{i}^{*}\right)$ (cf. (8)), where $P_{i}^{*}$ denotes the maximizer of (24). ${ }^{3}$ The following proposition presents sufficient conditions [9] for this approach to have the same error performance as optimal detector randomization:

Proposition 2: Define $P_{\max , i}=\max \left\{P_{\mathrm{av}, i}, P_{\mathrm{pk}, i}\right\}$. The problems in (21) and (24) achieve the same maximum value if at least one of the conditions holds:

- $\phi_{i}(P)$ is a concave function for $P \in\left[0, P_{\max , i}\right]$.

- $\quad \arg \max \phi_{i}(P) \leq \min \left\{P_{\mathrm{av}, i}, P_{\mathrm{pk}, i}\right\}$.

$P \in\left[0, P_{\max , i}\right]$

Detector Randomization Assuming Perfect Sensing: In this scenario, the secondary receiver assumes that the sensing decision is perfect (i.e., does not take channel sensing errors into account), and designs the optimal MAP detectors and the detector randomization factors according to the signal models in (1) and (4). ${ }^{4}$ (This approach is called suboptimal detector randomization in the following.)

Single Detector Assuming Perfect Sensing: In this case, the secondary receiver assumes that the sensing decision is perfect, and performs MAP detector design without any detector randomization; i.e., employs a single detector. (This approach is called suboptimal single detector in the following.)

Consider binary phase-shift keying (BPSK), where $d \in$ $\{-1,1\}$ with equal priors, and assume that the power levels are limited by the peak power constraint which is set as $P_{\mathrm{pk}, i}=3$ for $i \in\{0,1\}$. In Fig. 2, the average probabilities of error are plotted for the four approaches described above, where $\sigma^{2}=\sigma_{n}^{2}=\sigma_{j}^{2} \forall j$ in (22) and (23), and the parameters of the complex Gaussian mixture in (23) are given by $N_{m}=3, \boldsymbol{\mu}=\left[\begin{array}{lll}\mu_{1} & \mu_{2} & \mu_{3}\end{array}\right]=\left[\begin{array}{lll}-1 & 0 & 1\end{array}\right]$, and $\boldsymbol{\nu}=\left[\begin{array}{lll}\nu_{1} & \nu_{2} & \nu_{3}\end{array}\right]=\left[\begin{array}{lll}0.25 & 0.5 & 0.25\end{array}\right]$. Also, the average power limits $P_{\mathrm{av}, 0}$ and $P_{\mathrm{av}, 1}$ in (5) are set to $P_{\mathrm{av}, 0}=1.3$ and $P_{\text {av }, 1}=0.4$, and $\operatorname{Pr}\left\{\hat{\mathcal{H}}_{1} \mid \mathcal{H}_{1}\right\}=0.9$. In the figure, the

\footnotetext{
${ }^{3}$ For practical cases, $\min \left\{P_{\mathrm{av}, i}, P_{\mathrm{pk}, i}\right\}=P_{\mathrm{av}, i}$ in (24).

${ }^{4}$ The solution for this approach can be obtained similarly to Proposition 1 and (21), which is not presented here due to the space limitation.
}

TABLE I

SOLUTIONS FOR OPTIMAL SINGLE DETECTOR AND OPTIMAL DETECTOR RANDOMIZATION APPROACHES.

\begin{tabular}{|c|cc|cccccc|}
\hline $1 / \sigma^{2}$ & \multicolumn{3}{|c|}{ Single Detector } & \multicolumn{5}{|c|}{ Detector Randomization } \\
$(\mathrm{dB})$ & $P_{0}^{*}$ & $P_{1}^{*}$ & $\lambda_{0}^{*}$ & $P_{0,1}^{*}$ & $P_{0,2}^{*}$ & $\lambda_{1}^{*}$ & $P_{1,1}^{*}$ & $P_{1,2}^{*}$ \\
\hline 2 & 1.300 & 0.400 & 1 & 1.300 & N/A & 1 & 0.400 & N/A \\
6 & 1.300 & 0.400 & 1 & 1.300 & N/A & 1 & 0.400 & N/A \\
10 & 1.300 & 0.400 & 1 & 1.300 & N/A & 1 & 0.400 & N/A \\
14 & 1.300 & 0.400 & 0.062 & 0.818 & 1.332 & 0.395 & 0.079 & 0.610 \\
18 & 1.300 & 0.070 & 0.318 & 0.685 & 1.586 & 0.380 & 0.069 & 0.603 \\
22 & 0.617 & 0.065 & 0.315 & 0.616 & 1.614 & 0.354 & 0.065 & 0.583 \\
25 & 0.591 & 0.064 & 0.301 & 0.590 & 1.606 & 0.341 & 0.064 & 0.574 \\
\hline
\end{tabular}

average error probabilities are plotted both versus $1 / \sigma^{2}$ (for $\mathrm{P}_{\mathrm{f}}=\operatorname{Pr}\left\{\hat{\mathcal{H}}_{1} \mid \mathcal{H}_{0}\right\}=0.1$ ) and versus $\mathrm{P}_{\mathrm{f}}$ (for $\sigma=0.1$ ). Please note that the $\mathrm{X}$-axis labels are at the bottom and top for $1 / \sigma^{2}$ and $\mathrm{P}_{\mathrm{f}}$, respectively. It is observed that the optimal detector randomization approach achieves the lowest average probabilities of error among all the approaches for all $\mathrm{P}_{\mathrm{f}}$ and for reasonably low values of $\sigma^{2}$ (namely, when $1 / \sigma^{2}$ is larger than $10 \mathrm{~dB}$, which correspond to practical error rates.) Also, the optimal single detector and suboptimal detector randomization algorithms can have various amounts of improvements over the suboptimal single detector approach in different scenarios (e.g., $1 / \sigma^{2}=16 \mathrm{~dB}$ and $1 / \sigma^{2}=22 \mathrm{~dB}$ ). It is also concluded that improvements obtained via the consideration of possible sensing errors become significant in the presence of high sensing error probability (high $\mathrm{P}_{\mathrm{f}}$ in this example).

In Table I, the solutions of the optimal single detector and optimal detector randomization approaches are presented for the scenario in Fig. 2 (i.e., for $\mathrm{P}_{\mathrm{f}}=0.1$ ). The solution of the optimal single detector approach, which is obtained from (24), is denoted by $P_{0}^{*}$ and $P_{1}^{*}$, which correspond to the optimal power levels employed when the sensing decision is $\hat{\mathcal{H}}_{0}$ and $\hat{\mathcal{H}}_{1}$, respectively. On the other hand, the solution of the optimal detector randomization approach, calculated from (21), is expressed by $\lambda_{i}^{*}, P_{i, 1}^{*}$, and $P_{i, 2}^{*}$ for $i \in\{0,1\}$ (please see (20)). That is, when the sensing decision is $\hat{\mathcal{H}}_{i}$, the optimal detector randomization approach employs power levels $P_{i, 1}^{*}$ and $P_{i, 2}^{*}$ for $\lambda_{i}^{*}$ and $\left(1-\lambda_{i}^{*}\right)$ fractions of time, respectively, with the corresponding MAP detectors. From the table, it is observed that the two approaches result in the same solution for large $\sigma$ values.

\section{REFERENCES}

[1] T. Yucek and H. Arslan, "A survey of spectrum sensing algorithms for cognitive radio applications," IEEE Commun. Surveys and Tutorials, vol. 11, no. 1, pp. 116-130, 2009.

[2] M. C. Gursoy and S. Gezici, "On the interplay between channel sensing and estimation in cognitive radio systems," in Proc. 2011 IEEE Global Telecommun. Conf.

[3] S. Stotas and A. Nallanathan, "Overcoming the sensing-throughput tradeoff in cognitive radio networks," in Proc. 2010 IEEE Int. Conf. on Commun.

[4] A. Patel and B. Kosko, "Optimal noise benefits in Neyman-Pearson and inequality-constrained signal detection," IEEE Trans. Signal Process., vol. 57, no. 5, pp. 1655-1669, May 2009.

[5] B. Dulek and S. Gezici, "Detector randomization and stochastic signaling for minimum probability of error receivers," IEEE Trans. Commun., vol. 60, no. 4, pp. 923-928, Apr. 2012.

[6] S. Bayram, S. Gezici, and H. V. Poor, "Noise enhanced hypothesistesting in the restricted Bayesian framework," IEEE Trans. Signal Process., vol. 58, no. 8, pp. 3972-3989, Aug. 2010.

[7] R. T. Rockafellar, Convex Analysis. Princeton University Press, 1968.

[8] F. Moghimi, A. Nasri, and R. Schober, "Adaptive $l_{p}$-norm spectrum sensing for cognitive radio networks," IEEE Trans. Commun., vol. 59, no. 7, pp. 1934-1945, July 2011.

[9] H. Soganci, S. Gezici, and O. Arikan, "Optimal stochastic parameter design for estimation problems," IEEE Trans. Signal Process., vol. 60, no. 9, pp. 4950-4956, Sep. 2012. 